Check for updates

Cite this: Phys. Chem. Chem. Phys., 2019, 21, 11651

Received 31st March 2019. Accepted 10th May 2019

DOI: $10.1039 / c 9 c p 01802 f$

rsc.li/pccp

\section{Vibrational spectroscopy of the hexahydrated sulfate dianion revisited: role of isomers and anharmonicities $\dagger$}

\begin{abstract}
Harald Knorke, ${ }^{a}$ Huiyan Li, ${ }^{\text {bc }}$ Zhi-Feng Liu*bc and Knut R. Asmis (D) *a
We report on the gas phase vibrational spectroscopy of the hexahydrated sulfate dianion, $\mathrm{SO}_{4}{ }^{2-}\left(\mathrm{H}_{2} \mathrm{O}\right)_{6}$, and its fully deuterated isotopologue, $\mathrm{SO}_{4}{ }^{2-}\left(\mathrm{D}_{2} \mathrm{O}\right)_{6}$, using infrared photodissociation (IRPD) spectroscopy of the $D_{2}$-tagged dianions in combination with density-functional-theory calculations on minimumenergy structures as well as finite temperature ab initio molecular dynamics (AIMD) simulations. The IRPD spectra were recorded at an ion trap temperature of $12 \mathrm{~K}$ and in the spectral range from 650 to $3800 \mathrm{~cm}^{-1}$, covering the intramolecular modes of the solvent $\left(\mathrm{OH} / \mathrm{OD}\right.$ stretches and $\mathrm{H}_{2} \mathrm{O} / \mathrm{D}_{2} \mathrm{O}$ bends) at higher energies, those of the solute (sulfate stretches) at intermediate energies and the intermolecular solute librational modes at the lowest energies. Isomer-specific double resonance in combination with messenger-tag dependent IRPD spectra show that only a single isomer is contributing significantly and that this isomer is not the highly symmetric $T_{d}$ but rather the lower symmetry $C_{3}$ isomer. Temperaturedependent IR multiple photon dissociation spectra of bare $\mathrm{SO}_{4}{ }^{2-}\left(\mathrm{H}_{2} \mathrm{O}\right)_{6}$ suggest that the $C_{3}$ isomer remains the most stable one up to $200 \mathrm{~K}$. The AIMD simulations reveal that the IRPD spectra can only be fully understood when anharmonic effects as well as entropy-driven hydrogen bond network fluctuations are considered.
\end{abstract}

\section{Introduction}

Hydrated sulfate dianions, $\mathrm{SO}_{4}{ }^{2-}$, play a key role in the homogeneous nucleation of ice particles by sulfate aerosols in the upper troposphere ${ }^{1}$ and in the regulation of many metabolic and cellular processes. ${ }^{2}$ The presence of water on the Martian surface has been inferred from sulfate geochemistry. ${ }^{3}$ Due to its compact and doubly charged nature, the interaction of sulfate with water is strong and sulfate adopts a distinct position in the famous Hofmeister series as one of the most kosmotropic anions. ${ }^{4}$ Microhydrated sulfate dianions in the gas phase serve as prototypical model systems for understanding anion solvation, in general, and that of multiply-charged anions, in particular. ${ }^{5,6}$ The structure of the hexahydrated sulfate dianion has been the subject of ongoing discussions over the last two decades. $^{7-15}$ Two low-energy structures exist (see Fig. 1), which

\footnotetext{
${ }^{a}$ Wilhelm-Ostwald-Institut für Physikalische und Theoretische Chemie, Universität Leipzig, Linnéstr. 2, 04103 Leipzig, Germany.E-mail: knut.asmis@uni-leipzig.de ${ }^{b}$ Department of Chemistry and Centre for Scientific Modelling and Computation, Chinese University of Hong Kong, Hong Kong, China. E-mail: zfliu@cuhk.edu.hk ${ }^{c}$ Shenzhen Research Institute, Chinese University of Hong Kong,

No. 10, 2nd Yuexing Road, Shenzhen, China

$\dagger$ Electronic supplementary information (ESI) available: Mass spectrum, relative electronic energies and Gibbs free energies, simulated harmonic and anharmonic IR spectra, fit parameters. See DOI: $10.1039 / \mathrm{c} 9 \mathrm{cp} 01802 \mathrm{f}$
}

differ in the way the six water molecules function as hydrogenbond acceptors (A) and donors (D). The $C_{3}$ isomer contains two types of water molecules, three DD and three ADD ones, and is referred to as the 6.9.3 isomer, since it contains six water molecules that form nine anion-water and three water-water hydrogen bonds. ${ }^{13}$ In contrast, the six DD water molecules in the $T_{\mathrm{d}}$ isomer $\mathbf{6 . 1 2 . 0}$ are symmetry equivalent, forming twelve anion-water and no water-water hydrogen bonds. Here, we study the vibrational spectroscopy of the hexahydrated sulfate dianions, $\mathrm{SO}_{4}{ }^{2-}\left(\mathrm{H}_{2} \mathrm{O}\right)_{6}$, and its fully deuterated isotopologue, $\mathrm{SO}_{4}{ }^{2-}\left(\mathrm{D}_{2} \mathrm{O}\right)_{6}$, in order to unambiguously resolve, which isomers contribute to its gas phase IR spectrum at cryogenic temperatures and above.

Blades and Kebarle ${ }^{5}$ were the first to detect $\mathrm{SO}_{4}{ }^{2-}\left(\mathrm{H}_{2} \mathrm{O}\right)_{n}$ dianions with $n \geq 3$ in the gas phase and subsequently determined some of their hydration energies. ${ }^{16}$ Wang and co-workers performed the first spectroscopic investigations using anion photoelectron (APE) spectroscopy and suggested that the signal carrier is the $C_{3}$ isomer 6.9.3 (see Fig. 1), based on density functional theory (DFT) calculations. ${ }^{7}$ Subsequently, second-order Møller-Plesset (MP2) calculations by Pye and Rudolph identified the $T_{\mathrm{d}}$ isomer 6.12.0 (see Fig. 1) as the global minimum. ${ }^{8}$ Computational studies that directly followed provided evidence for several energetically low-lying isomers and could not convincingly resolve this issue. ${ }^{9}$ The first gas 

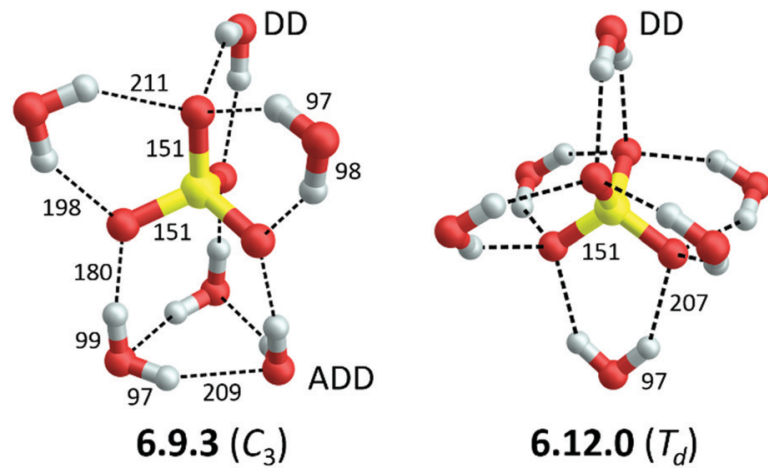

6.12.0 $\left(T_{d}\right)$

Fig. 1 B3LYP-D3/aug-cc-pVTZ minimum-energy structures, including label (see text and ref. 7 for details) and symmetry, of the two lowest energy structures of $\mathrm{SO}_{4}{ }^{2-}\left(\mathrm{H}_{2} \mathrm{O}\right)_{6}$. Bond lengths are reported in pm. Water molecules are classified according to their function as hydrogen bond acceptor (A) or donor (D)

phase vibrational spectra, in the form of infrared multiple photon photodissociation (IRMPD) spectra of cryogenicallycooled ions at $\sim 20 \mathrm{~K}$ in the mid-IR range (550 to $1800 \mathrm{~cm}^{-1}$ ) using the widely-tunable IR radiation from a free electron laser were published in 2006. ${ }^{10}$ Comparison to simulated harmonic IR spectra from MP2 calculations yielded slightly better agreement with the $T_{\mathrm{d}}$ isomer 6.12.0 in this spectral range. Shortly thereafter, Williams and co-workers measured an IRMPD spectrum of slightly hotter anions $(130 \mathrm{~K})$, but now in the $\mathrm{O}-\mathrm{H}$ stretching region, and came to the conclusion that multiple isomers, in particular the $C_{3}$ and $T_{\mathrm{d}}$ isomers, are present and contribute to the IRMPD spectra. ${ }^{11}$ APE experiments on cryogenically cooled dianions at $12 \mathrm{~K}$, which allow for a more accurate determination of the vertical detachment energy, combined with higher level calculations, on the other hand, confirmed the initial assignment by Wang et al. to the $C_{3}$ isomer. ${ }^{12}$ The authors also noted that at room temperature multiple isomers should be populated. Head-Gordon, Xantheas and co-workers performed the first coupled cluster calculations, also in combination with anharmonic vibrational corrections and estimates of the basis set effects up to the complete basis set limit using MP2, on this system. ${ }^{13,14}$ Interestingly, these calculations predict yet another structure, a 6.6.6 structure containing two three-membered water rings, to be the global minimum, but after zero-point energy corrections are taken into account the $C_{3}$ isomer (6.9.3) is predicted to be the most stable one at $0 \mathrm{~K}$ for both isotopologues. However, the authors also note that "the errors in the different theoretical treatments might still be too large to make a clear distinction between very closely spaced isomers possible". ${ }^{14}$ Since anharmonic as well as entropic effects are important for a reliable description of such systems a molecular dynamics description of this system may prove more appropriate. ${ }^{13,17}$

In this publication we consider many of the previously made suggestions and report messenger-tagged spectra of cold, hexahydrated sulfate dianions. We explicitly explore anharmonic effects by studying the effect of (i) isotope-substitution, (ii) the number of messenger tags and (iii) temperature on the vibrational spectra. Furthermore, we apply an established IR double-resonance detection scheme ${ }^{18}$ to identify the contributions to the vibrational spectra isomer-specifically. The interpretation of the experimental data is aided by the results from harmonic and anharmonic vibrational frequency calculations as well as $a b$ initio molecular dynamics (AIMD) simulations using DFT.

\section{Methods}

\section{Experimental methods}

IRPD experiments were performed using the $6 \mathrm{~K}$ ion trap triple mass spectrometer described previously. ${ }^{19}$ In brief, microhydrated sulfate dianions are produced in a nanospray ion source from a $20 \mathrm{mM}$ solution of sulfuric acid in a 1:2 water/methanol mixture. A typical mass spectrum of this solution is shown in Fig. S1 of the ESI. $\dagger$ The beam of anions is skimmed, collimated in a gas-filled radio frequency (RF) ion guide, mass-selected using a quadrupole mass-filter and focused in a RF ringelectrode ion trap, held at a temperature in-between 6 and $300 \mathrm{~K}$ using a closed-cycle helium cryostat. To allow for continuous ion loading and ion thermalization, the trap is continuously filled with buffer gas, either $\mathrm{He}$ (for bare ions) or $\mathrm{D}_{2}$ (for messenger-tagged ions). Many collisions of the trapped ions with the buffer gas provide gentle cooling of the internal degrees of freedom close to the ambient temperature. At sufficiently low ion-trap temperatures, ion-messenger complexes are formed via three-body collisions. ${ }^{20}$ Every $100 \mathrm{~ms}$, all ions are extracted from the ion trap and focused both temporally and spatially into the center of the extraction region of an orthogonally-mounted double-focussing reflectron time-offlight (TOF) tandem photofragmentation mass spectrometer. The ion packet is accelerated into the reflectron stage, ions spread out in space according to their mass-to-charge ratio $(\mathrm{m} / \mathrm{z})$ and are refocused at the initial extraction region. Prior to reacceleration towards the MCP detector, ions with a particular $\mathrm{m} / \mathrm{z}$ value are irradiated by a properly timed, widely wavelength tunable IR laser pulse (bandwidth: $2.5 \mathrm{~cm}^{-1}$ ), supplied by an optical parametric oscillator/amplifier (LaserVision: OPO/OPA/ $\mathrm{AgGaSe}_{2}$ ) laser system. ${ }^{21}$ An IRPD spectrum is measured by continuously scanning the laser wavelength, which is monitored online using a HighFinesse WS6-600 wavelength meter, with a scan speed such that an averaged TOF mass spectrum (over 150 laser shots) is obtained every $2 \mathrm{~cm}^{-1}$. Typically, at least three scans are measured and averaged and the photodissociation cross section $\sigma_{\text {IRPD }}$ is determined as described previously. ${ }^{19}$

\section{Computational methods}

Electronic structure calculations were performed using the Gaussian 16 rev. A.03 program. ${ }^{22}$ Previously reported geometries $^{10,13}$ were used as initial guesses for geometry optimizations followed by vibrational frequency calculations using the B3LYP functional ${ }^{23}$ in combination with the aug-cc-pVTZ ${ }^{24}$ basis set. The influence of dispersion interactions was considered 
by using the D3 version of Grimme's dispersion correction with Becke-Johnson damping. ${ }^{25}$ To account for anharmonic effects as well as systematic errors on the harmonic force constants, we determined scaling factors on the basis of a comparison of the IRPD spectra to harmonic spectra of both isotopologues (all-H and all-D). To assess these effects more carefully, we performed anharmonic frequency calculations using the second-order vibrational perturbation theory (VPT2) method as implemented by Bloino and Barone. ${ }^{26}$ Relevant optimized geometries, minimum energies and harmonic and anharmonic vibrational spectra are reported in the ESI. $\dagger$

To uniquely identify the numerous isomers, we adopt the labelling proposed by Lambrecht et al. ${ }^{13,14}$ The label of each individual $\mathrm{SO}_{4}{ }^{2-}\left(\mathrm{H}_{2} \mathrm{O}\right)_{n}$ structure is given in the $n . \mathrm{s} . \mathrm{w}-l$ notation, where $n, \mathrm{~s}$ and $\mathrm{w}$ refer to the number of water molecules, of sulfate-water hydrogen bonds and of water-water hydrogen bonds, respectively. Within each n.s.w category the structures are sorted according to increasing electronic energy determined by Lambrecht et al. ${ }^{14}$ and indicated with number $l(l=1,2, \ldots)$. Note, when $l$ is omitted $l=1$ is implied.

In AIMD simulations, the electronic energy and atomic forces are obtained within the framework of DFT, while the atomic motion is treated within Newtonian mechanics, as implemented in the CP2K package. ${ }^{27}$ The wave functions are expanded in a double zeta Gaussian basis set, while the electron density is expanded in Gaussians and auxiliary plane waves (GPW) ${ }^{28}$ with an energy cut-off at 350 Rydberg for the electron density. The atomic cores are modelled by the Goedecker-Teter-Hutter (GTH) type pseudopotentials. ${ }^{29}$ The exchange and correlation energy is calculated using the BLYP functional $^{30}$ in combination with Grimme's D3 dispersion correction. ${ }^{25}$ A cluster ion is put at the center of a periodic cubic box, and the effects of the periodic charge density images are corrected by the decoupling technique developed by Martyna and Tuckerman. ${ }^{31}$ The box length is 16 to $20 \AA$ depending on the cluster size. The convergence criterion for the SCF electronic procedure is set to be $10^{-7}$ a.u. at each time step. For molecular dynamics, the temperature is controlled by a Nose-Hoover thermostat, ${ }^{32}$ with a time step of 0.5 fs. An equilibration period of up to 15 ps is performed first, while a production run for data collection at $100 \mathrm{~K}$ lasts more than 100 ps.

A vibrational spectrum can be directly simulated by the Fourier transform of the dipole time-correlation function (DTCF), ${ }^{33}$

$$
(\omega)=\frac{2 \pi \beta \omega^{2}}{3 n(\omega) c V} \int_{-\infty}^{+\infty} \mathrm{d} t\langle\vec{M}(t) \cdot \vec{M}(0)\rangle \exp (\mathrm{i} \omega t)
$$

where $\beta=1 / k T, n(\omega)$ is the refractive index, $c$ the speed of light in vacuum, and $V$ the volume. $\vec{M}$ is the total dipole moment of the system, calculated by the polarization including both ionic and electronic contributions at each time step. The temperature and dynamic effects produced by the fluctuations of HBs on the vibrations are automatically captured by AIMD simulations and contained in such a DTCF spectrum.

\section{Results and discussion}

\section{IRPD spectra of the all-H and all-D isotopologues}

IRPD spectra of $\mathrm{D}_{2}$-tagged $\mathrm{SO}_{4}{ }^{2-}\left(\mathrm{H}_{2} \mathrm{O}\right)_{6}$ and $\mathrm{SO}_{4}{ }^{2-}\left(\mathrm{D}_{2} \mathrm{O}\right)_{6}$ in the $\mathrm{O}-\mathrm{H}(\mathrm{D})$ stretching and the mid-IR spectral regions are shown in Fig. 2. Band positions and assignments are summarized in Table 1 . The IRPD bands are labelled according to their assignment, i.e., either as hydrogen-bonded water stretching (a), $\mathrm{D}_{2}$ stretching (b), water bending (c), antisymmetric sulfate stretching (d) and water wagging (e) modes, numbered from highest to lowest energy. An apostrophe indicates the bands of the deuterated complex. For better comparability of the bands assigned to water modes, a scaling factor of 1.361 is used to align the $\mathrm{O}-\mathrm{H} / \mathrm{O}-\mathrm{D}$ stretching and $\mathrm{H}_{2} \mathrm{O} / \mathrm{D}_{2} \mathrm{O}$ bending regions. The scaling factor is close to the value expected for isolated $\mathrm{O}-\mathrm{H} v s$. $\mathrm{O}-\mathrm{D}$ oscillators (1/1.374). Note, non-water modes, like $\mathrm{SO}_{4}{ }^{2-}$ and $\mathrm{D}_{2}$ stretches (indicated by the broken lines in Fig. 2), which are centred at similar wavenumbers in the spectra of the two isotopologues, are not aligned in this representation.

Taking into account the expected isotopic shifts, the IRPD spectra of the two isotopologues shown in Fig. 2 look very similar, indicating that the same isomer (or isomers) is (are) probed in both cases. The spectra show no sign of free $\mathrm{O}-\mathrm{H}$ $\left(>3650 \mathrm{~cm}^{-1}\right)$ or $\mathrm{O}-\mathrm{D}\left(>2700 \mathrm{~cm}^{-1}\right)$ stretching modes, but a rather complicated pattern of at least eight (five) bands in the hydrogen-bonded $\mathrm{O}-\mathrm{H}(\mathrm{D})$ stretching region extending from 3600 to $3100 \mathrm{~cm}^{-1}$ (2650 to $\left.2300 \mathrm{~cm}^{-1}\right)$. This characteristic IR signature in the hydrogen-bonded $\mathrm{O}-\mathrm{H}(\mathrm{D})$ stretching region is similarly broad to that observed previously by Bush et al. in their IRMPD study. ${ }^{11}$ This supports their previous assignment to a lower-symmetry isomer containing hydrogen-bonded $\mathrm{O}-\mathrm{H}(\mathrm{D})$ oscillators in various environments, i.e. exhibiting a broader distribution of red-shifted $\mathrm{O}-\mathrm{H}$ stretching transitions, like the 6.9.3 isomer, which contains weak (water-water), intermediate (DD water-sulfate) and stronger (ADD water-sulfate) hydrogen bonds. The highly symmetric $T_{\mathrm{d}}$ isomer $\mathbf{6 . 1 2 . 0}$, on the other hand, exhibits only a single type of hydrogen bonded O-H(D) oscillator (DD water-sulfate) and consequently should yield a less red-shifted and much narrower absorption profile.

In more detail, both spectra contain three intense bands at $3559 / 2625 \mathrm{~cm}^{-1}\left(\mathrm{a}_{1} / \mathrm{a}_{1}{ }^{\prime}\right), 3457 / 2546 \mathrm{~cm}^{-1}\left(\mathrm{a}_{3} / \mathrm{a}_{3}{ }^{\prime}\right)$ and $3172 /$ $2371 \mathrm{~cm}^{-1}\left(\mathrm{a}_{8} / \mathrm{a}_{8}{ }^{\prime}\right)$, which we assign, in agreement with previous calculations, ${ }^{10-14}$ to the stretching modes involving the predominantly decoupled $\mathrm{O}-\mathrm{H} /(\mathrm{D})$ oscillators of the ADD waters hydrogenbonded to either the adjacent ADD water molecules $\left(a_{1} / a_{1}{ }^{\prime}\right)$ or to the sulfate core $\left(\mathrm{a}_{8} / \mathrm{a}_{8}{ }^{\prime}\right)$ and the symmetric combination of coupled $\mathrm{O}-\mathrm{H}(\mathrm{D})$ oscillators of the DD waters $\left(\mathrm{a}_{3} / \mathrm{a}_{3}{ }^{\prime}\right)$. Note, while the band position ratios $\mathrm{a}_{1} / \mathrm{a}_{1}{ }^{\prime}$ (1.356) and $\mathrm{a}_{3} / \mathrm{a}_{3}{ }^{\prime}$ (1.358) are nearly identical, the ratio $\mathrm{a}_{8} / \mathrm{a}_{8}{ }^{\prime}(1.338)$ is smaller, indicating a larger anharmonicity in the all- $\mathrm{H}$ isotopologue of the latter mode and hence a less pronounced red-shift of $\mathrm{a}_{8}{ }^{\prime}$ (compared to $\mathrm{a}_{1}{ }^{\prime}$ and $\mathrm{a}_{3}{ }^{\prime}$, see Fig. 2) as a result of the lower zero-point energy (ZPE) in the all-D isotopologue. The weaker features $\mathrm{a}_{4-7}$ are attributed to combination and overtone excitations. The band position ratio $a_{2} / a_{2}{ }^{\prime}(1.354)$ is similar to that of $a_{1} / a_{1}{ }^{\prime}$, while features $a_{4-7}$ seem to exhibit a similar behaviour to $a_{8}$, 


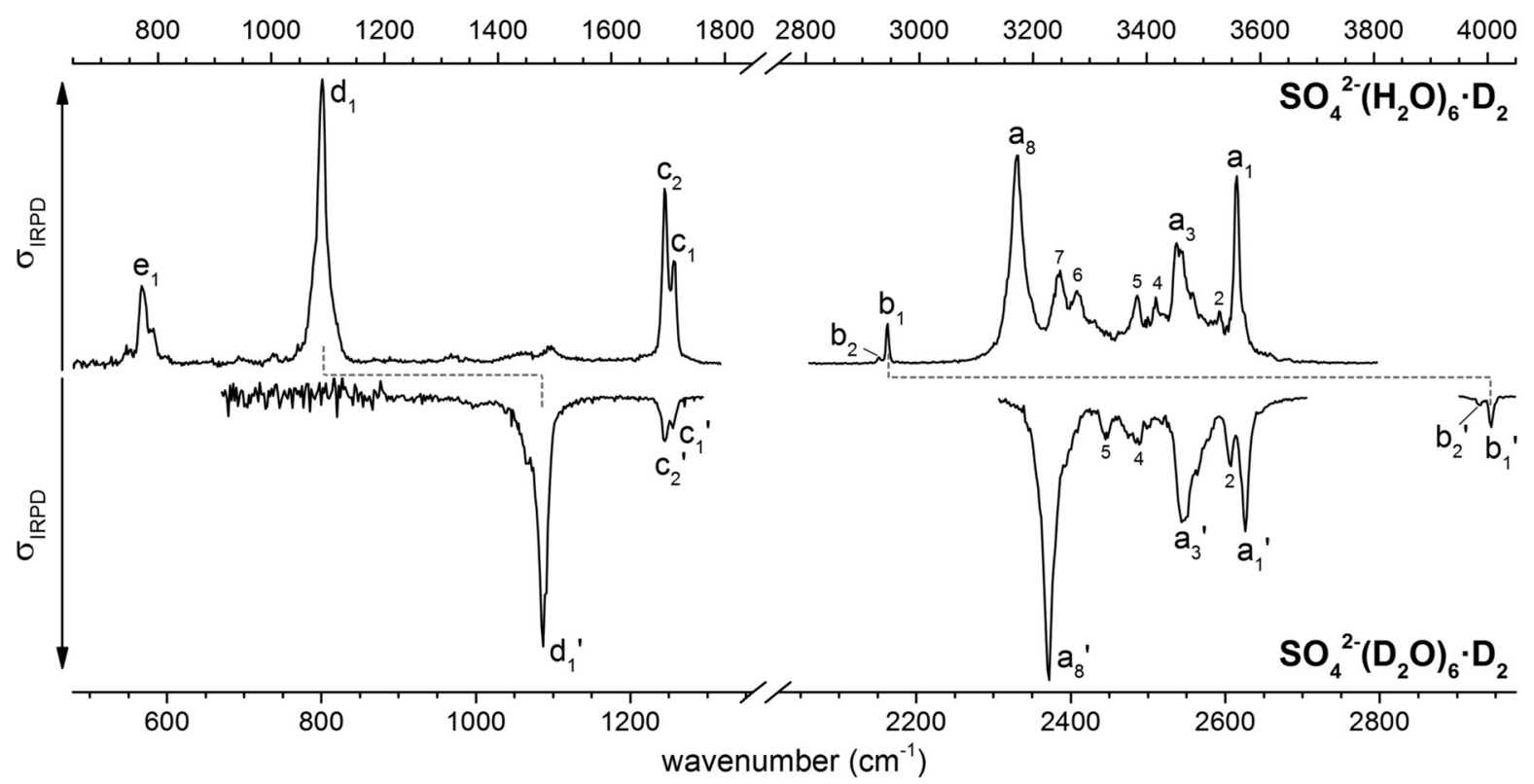

Fig. 2 IRPD spectra of the $\mathrm{D}_{2}$-tagged all-H and all-D isotopologues of the hexahydrated sulfate dianion: $\mathrm{SO}_{4}{ }^{2-}\left(\mathrm{H}_{2} \mathrm{O}\right)_{6} \cdot \mathrm{D}_{2}(\mathrm{top})$ and $\mathrm{SO}_{4}{ }^{2-}\left(\mathrm{D}_{2} \mathrm{O}\right)_{6} \cdot \mathrm{D}_{2}$ (bottom). Note, for better comparability the spectra are plotted against opposing vertical axes and the horizontal axis of the lower spectrum was scaled 1/1.361, such that the bands corresponding water modes, i.e. bands $a / a^{\prime}$ and $c / c^{\prime}$ appear at similar horizontal positions (see text). See Table 1 for band labels, positions and assignments.

suggesting that this mode $\left(\mathrm{a}_{8}\right)$ contributes to some of these combination bands. In addition, the intensities of nonfundamental transition are markedly suppressed in the all-D spectrum, in agreement with smaller anharmonicities and hence less strong couplings with low-energy modes in the all-D isotopologue.

The nominally IR-forbidden D-D stretching mode of the messenger tag, which gains IR-activity upon polarization, is observed red-shifted $\left(50-65 \mathrm{~cm}^{-1}\right)$ from its value in free $D_{2}$ $\left(2994 \mathrm{~cm}^{-1}\right) \cdot{ }^{34}$ Interestingly, this region exhibits more than one peak, an intense one at $2944 \mathrm{~cm}^{-1}\left(\mathrm{~b}_{1}, \mathrm{~b}_{1}{ }^{\prime}\right)$ and a roughly seven (four) times weaker one at $2929 \mathrm{~cm}^{-1}\left(\mathrm{~b}_{2}, \mathrm{~b}_{2}{ }^{\prime}\right)$, suggesting more than one $\mathrm{D}_{2}$ binding site. Note, three type of $\mathrm{D}_{2}$ binding sites (see below) are present in the 6.9.3 isomer, while the 6.12.0 isomer exhibits only a single type.

A pair of peaks, at $1710 \mathrm{~cm}^{-1}\left(\mathrm{c}_{1}\right)$ and $1694 \mathrm{~cm}^{-1}\left(\mathrm{c}_{2}\right)$ in the all-H and at $1256 \mathrm{~cm}^{-1}\left(\mathrm{c}_{1}{ }^{\prime}\right)$ and $1245 \mathrm{~cm}^{-1}\left(\mathrm{c}_{2}{ }^{\prime}\right)$ in the all-D spectrum, are observed in the water bending region. These bands exhibit the same band position ratios $\left(\mathrm{c}_{1} / \mathrm{c}_{1}{ }^{\prime}: 1.361\right.$, $\left.\mathrm{c}_{2} / \mathrm{c}_{2}{ }^{\prime}: 1.361\right)$ as well as similar band intensity ratios $\left(\mathrm{c}_{1} / \mathrm{c}_{2}\right.$ : $\left.59 \%, c_{1}{ }^{\prime} / c_{2}{ }^{\prime}: 71 \%\right)$. This suggests a similar number of water molecules in two type of binding environments, again

Table 1 Experimental band positions, unscaled B3LYP-D3/aug-cc-pVTZ harmonic vibrational wavenumbers (both in $\mathrm{cm}^{-1}$ ) and assignments of the features observed in the IRPD of $\mathrm{D}_{2}$-tagged $\mathrm{SO}_{4}{ }^{2-}\left(\mathrm{H}_{2} \mathrm{O}\right)_{6}$ and $\mathrm{SO}_{4}{ }^{2-}\left(\mathrm{D}_{2} \mathrm{O}\right)_{6}$ and in the IRMPD of $\mathrm{SO}_{4}{ }^{2-}\left(\mathrm{H}_{2} \mathrm{O}\right)_{6}$

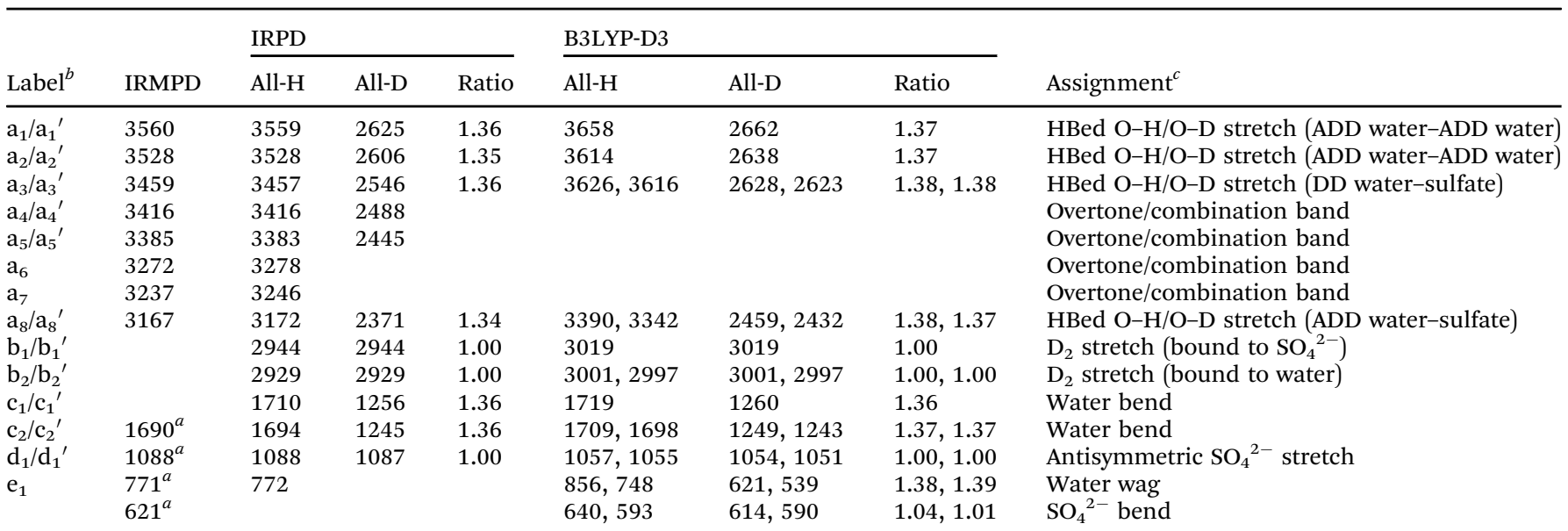

${ }^{a}$ Ref. $14 .{ }^{b}$ IRPD bands associated with the all-D isotopomer are labelled with an apostrophe in Fig. $2 .{ }^{c}$ Abbreviations: hydrogen-bonded (HBed), donor-donor (DD), acceptor-donor-donor (ADD, see text). 
favouring an assignment to the 6.9.3 isomer, which contains three DD and three ADD waters, rather than to the 6.12.0 isomer with its six symmetry-equivalent DD waters.

A single peak $\mathrm{d}_{1}\left(\mathrm{~d}_{1}{ }^{\prime}\right)$ with a width of $23 \mathrm{~cm}^{-1}\left(17 \mathrm{~cm}^{-1}\right)$ is observed at $1088 \mathrm{~cm}^{-1}\left(1087 \mathrm{~cm}^{-1}\right)$ in the sulfate stretching region of the IRPD spectra of the all-H (all-D) isotopologue. The absence of any significant splitting (considering the experimental resolution of a few $\mathrm{cm}^{-1}$ ) of the antisymmetric sulfate stretching mode suggest that the microhydration environment does not significantly perturb the triply degenerate transition, in agreement with the predictions for both isomers. ${ }^{12}$ However, bands $\mathrm{d}_{1}$ and $\mathrm{d}_{1}{ }^{\prime}$ do have slightly different band shapes: band $\mathrm{d}_{1}$ is more symmetrically broadened at its base, while $\mathrm{d}_{1}{ }^{\prime}$ is narrower, presumably due to the smaller rotational constants of the all-D isotopologue, and asymmetric.

\section{Isomer-specific $\mathrm{IR}^{2} \mathrm{MS}^{2}$ spectra}

In order to determine how many different isomers contribute to the IRPD spectrum of $\mathrm{SO}_{4}{ }^{2-}\left(\mathrm{H}_{2} \mathrm{O}\right)_{6} \cdot \mathrm{D}_{2}$ we performed isomer-selective $\mathrm{IR}^{2} \mathrm{MS}^{2}$ measurements. ${ }^{28,29}$ More precisely, the method is sensitive to laser-induced population changes that are maintained longer than the delay between the two IR laser pulses $\left(\sim 10^{-5} \mathrm{~s}\right)$. The IR spectra of more quickly interconverting isomers cannot be isolated using this technique. $\mathrm{IR}^{2} \mathrm{MS}^{2}$ spectra probed in $\mathrm{O}-\mathrm{H}$ oscillator region (3800-2900 $\left.\mathrm{cm}^{-1}\right)$ at $3559 \mathrm{~cm}^{-1}\left(\mathrm{a}_{1}\right), 3457 \mathrm{~cm}^{-1}\left(\mathrm{a}_{3}\right)$ and $3172 \mathrm{~cm}^{-1}\left(\mathrm{a}_{8}\right)$ are shown in Fig. 3 along with the (singleresonance) IRPD spectrum from Fig. 2. The three $\mathrm{IR}^{2} \mathrm{MS}^{2}$ spectra look very similar and thus provide strong evidence for the predominant presence of only a single isomer under these experimental conditions.
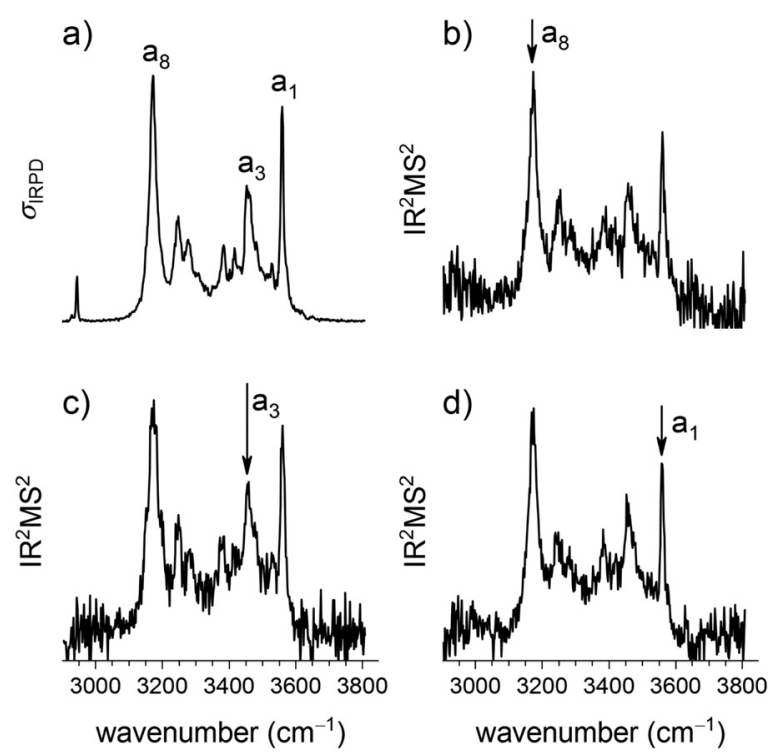

Fig. 3 Comparison of the (single resonance) IRPD spectrum of $\mathrm{SO}_{4}{ }^{2-}\left(\mathrm{H}_{2} \mathrm{O}\right)_{6} \cdot \mathrm{D}_{2}$ (a) from Fig. 2 to isomer-specific double-resonance $I^{2} \mathrm{MS}^{2}$ spectra probed at $3172 \mathrm{~cm}^{-1}$ (b), $3457 \mathrm{~cm}^{-1}$ (c) and $3559 \mathrm{~cm}^{-1}$ (d), corresponding to bands $a_{8}, a_{3}$ and $a_{1}$, respectively, each marked by an arrow.

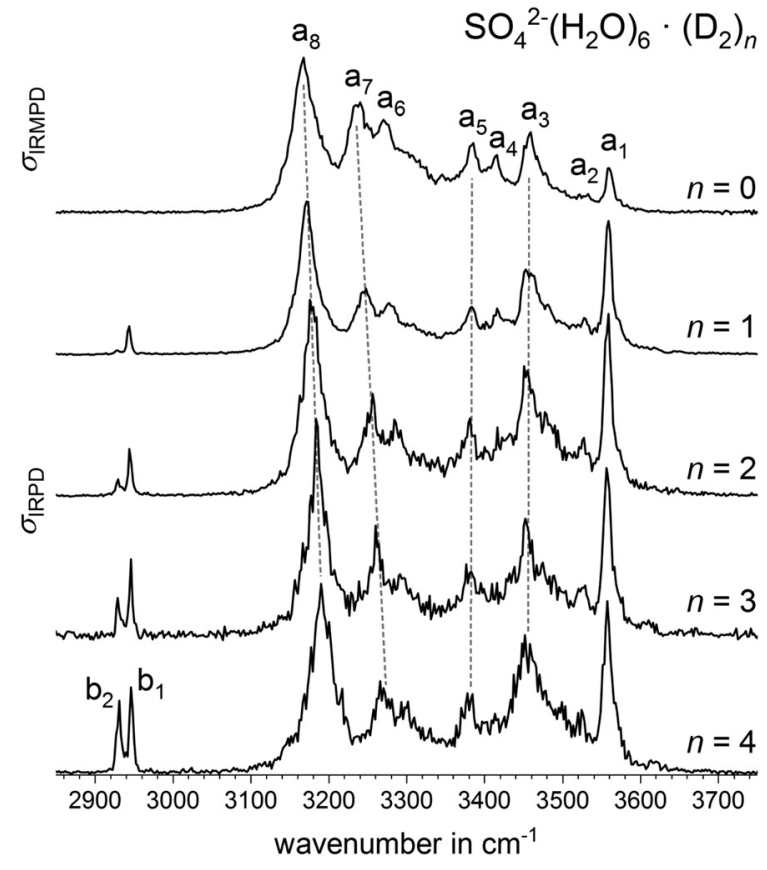

Fig. 4 Comparison of the IRMPD spectrum of $\mathrm{SO}_{4}{ }^{2-}\left(\mathrm{H}_{2} \mathrm{O}\right)_{6}$ (top trace) to IRPD spectra of the $\mathrm{D}_{2}$-tagged clusters $\mathrm{SO}_{4}{ }^{2-}\left(\mathrm{H}_{2} \mathrm{O}\right)_{6} \cdot\left(\mathrm{D}_{2}\right)_{n}$ with $n=1-4$ measured at an ion trap temperature of $12 \mathrm{~K}$.

\section{Influence of the messenger tag}

Additional information regarding the identity of the isomer can be obtained by comparing the IRPD spectra of complexes containing a different number of $\mathrm{D}_{2}$ tags $n$ (see Fig. 4). The spectra were measured in the $\mathrm{O}-\mathrm{H}$ stretching region and an ion trap temperature of $12 \mathrm{~K}$. While the overall appearance of the spectra is similar, two apparent messenger-tag dependent effects are observed. First, the lower energy bands shift to higher energies as $n$ increases. This effect is most pronounced for band $\mathrm{a}_{8}$ and amounts to $\sim 6 \mathrm{~cm}^{-1}$ per $\mathrm{D}_{2}$ molecule. Secondly, the relative intensities of the two $\mathrm{D}_{2}$ stretches $\left(b_{1}, b_{2}\right)$ increase and change their ratio from roughly 9:1 to $6: 5$ with increasing $n$ (see Fig. S6 and Table S2, ESI $\dagger$ ). In more detail, $D_{2}$ binding to the site corresponding to $b_{1}$ is initially favoured, but saturation leads to binding of additional messenger tags to the other binding site $\left(b_{2}\right)$. Both these observations support an assignment to a lower symmetry isomer. Isomer 6.9.3, indeed, has three types of $\mathrm{D}_{2}$ binding sites, the O-atoms of the three DD waters and the three ADD waters as well as three undercoordinated O-atoms of $\mathrm{SO}_{4}{ }^{2-}$. Our DFT calculations suggest that adsorption at the $\mathrm{DD}-\mathrm{H}_{2} \mathrm{O}$ and $\mathrm{SO}_{4}{ }^{2-}$ sites is slightly favoured energetically and results in the blue shift of band $a_{8}$ that is apparent in Fig. 4 .

\section{Temperature-dependent IRMPD spectra}

Fig. 5 shows the comparison of the IRPD spectrum of $\mathrm{D}_{2}$-tagged $\mathrm{SO}_{4}{ }^{2-}\left(\mathrm{H}_{2} \mathrm{O}\right)_{6}$ measured at an ion trap temperature of $12 \mathrm{~K}$, with IRMPD spectra of bare $\mathrm{SO}_{4}{ }^{2-}\left(\mathrm{H}_{2} \mathrm{O}\right)_{6}$ for temperatures between 6 and $200 \mathrm{~K}$ in the $\mathrm{O}-\mathrm{H}$ stretching region. The IRMPD spectrum at $6 \mathrm{~K}$ shows the same number peaks as the IRPD spectrum of 


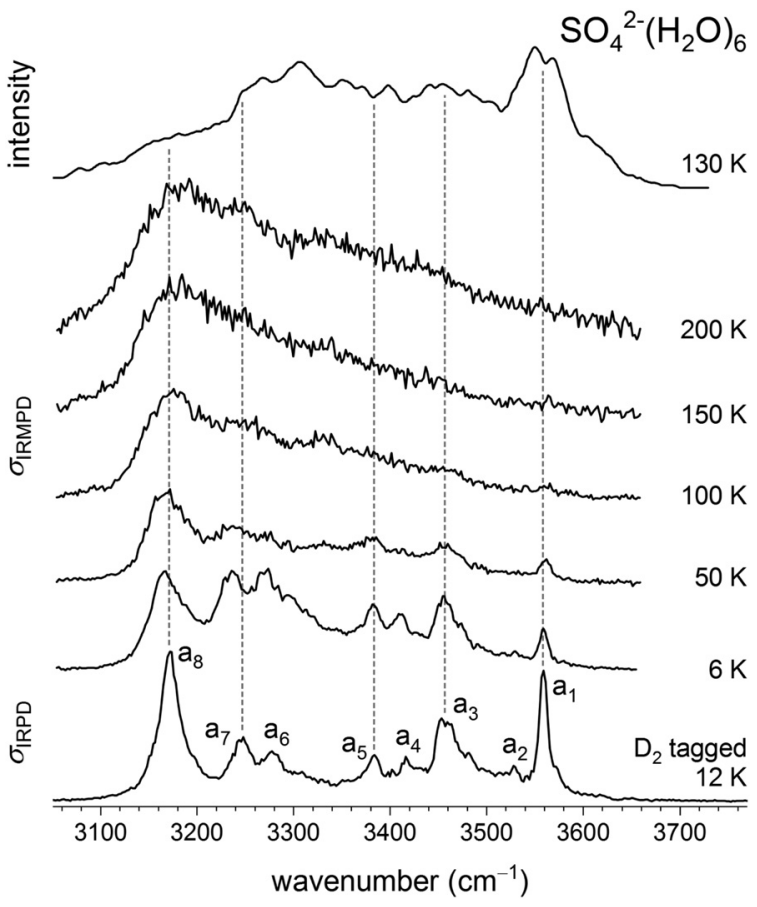

Fig. 5 IRMPD spectra of $\mathrm{SO}_{4}{ }^{2-}\left(\mathrm{H}_{2} \mathrm{O}\right)_{6}$ for ion trap temperatures from 6 to $200 \mathrm{~K}$. The $\mathrm{D}_{2}$-tagged IRPD spectrum measured at $12 \mathrm{~K}$, as well as, the spectrum measured by Williams and coworkers ${ }^{13}$ is also shown at the top.

the $\mathrm{D}_{2}$-tagged species, indicating that the same isomer is probed. However, the relative intensities of some of the peaks are different, in particular, those at higher energies, like those of $\mathrm{a}_{1}$ are smaller and those of the bands at lower energies, like $\mathrm{a}_{6}$ and $\mathrm{a}_{7}$, are larger in the IRMPD spectrum of untagged $\mathrm{SO}_{4}{ }^{2-}\left(\mathrm{H}_{2} \mathrm{O}\right)_{6}$. Note, photodissociation of bare $\mathrm{SO}_{4}{ }^{2-}\left(\mathrm{H}_{2} \mathrm{O}\right)_{6}$ (at $0 \mathrm{~K}$ ) requires at least two IR photons in this spectral region in order to overcome the predicted dissociation limit of $\sim 70 \mathrm{~kJ} \mathrm{~mol}{ }^{-1} .{ }^{14}$ We attribute the low intensity of $\mathrm{a}_{1}$ to less efficient IRMPD compared to the more anharmonic $\mathrm{O}-\mathrm{H}$ oscillators further to the red. Practically, the situation is more complex and mode-dependent details need to be considered. With increasing temperature the spectral features widen and merge into a single broad, asymmetric absorption band with a maximum around $3150 \mathrm{~cm}^{-1}$ at approximately $100 \mathrm{~K}$. Note, at higher energies the form of this absorption feature remains the same, providing no evidence for the population of additional isomers. This is significant, since the IRMPD spectrum reported by Williams and co-workers, ${ }^{11}$ obtained at $130 \mathrm{~K}$ and under different experimental conditions, lies in the same spectral region, but is more structured, with a strong absorption band at $3550 \mathrm{~cm}^{-1}$, which was attributed to the $\mathbf{6 . 1 2 . 0}$ isomer. We observe no such feature up to $200 \mathrm{~K}$, suggesting that this isomer is not populated thermally in our experiment. Hence, the different time scales in the two experiments lead to different isomer populations. In the present experiment the ions are irradiated by a single IR laser pulse $(12 \mathrm{~mJ})$ of $\sim 5 \mathrm{~ns}$ duration and this leads to the detection of only a single isomer (6.9.3), suggesting that isomerization is inefficient on this time scale. In the previous experiment the ions are irradiated by
Table 2 Relative Gibbs free energy $\left(\mathrm{kJ} \mathrm{mol}^{-1}\right)$ for the two relevant isomers and isotopologues. Electronic energies ${ }^{a}$ and harmonic vibrational energies were calculated at CCSD(T)/CBS* and B3LYP-D3/aug-cc-pVTZ level of theory, respectively

\begin{tabular}{|c|c|c|c|c|c|c|c|c|}
\hline \multirow[b]{2}{*}{ Isomer $^{c}$} & & \multicolumn{7}{|c|}{ Temperature (K) } \\
\hline & & 0 & 50 & 100 & 150 & 200 & 250 & 300 \\
\hline \multirow[t]{2}{*}{ All-H } & 6.12 .0 & 5.5 & 5.8 & 5.3 & 4.5 & 3.3 & 1.7 & 0.0 \\
\hline & 6.9 .3 & 0.0 & 0.0 & 0.0 & 0.0 & 0.0 & 0.0 & 0.0 \\
\hline \multirow[t]{2}{*}{ All-D } & 6.12 .0 & 7.7 & 7.9 & 7.3 & 6.1 & 4.5 & 2.7 & 0.6 \\
\hline & 6.9 .3 & 0.0 & 0.0 & 0.0 & 0.0 & 0.0 & 0.0 & 0.0 \\
\hline
\end{tabular}

70 to 1200 IR laser pulses ( $10 \mathrm{~Hz}, 8-21 \mathrm{~mJ})$, which gives them up to $120 \mathrm{~s}$ to isomerize (and/or radiatively decay) after the first photon is absorbed. In this way, the 6.12.0 isomer as well as others could be efficiently populated. Gibbs free energy calculations (see Table 2) show that the all-H (all-D) isotopologue of the 6.9.3 isomer is favoured by $6 \mathrm{~kJ} \mathrm{~mol}^{-1}\left(8 \mathrm{~kJ} \mathrm{~mol}^{-1}\right)$ over the 6.12.0 isomer at $0 \mathrm{~K}$. However, the higher symmetric (!) 6.12.0 isomer is entropically favoured at higher temperatures and its population is predicted to become relevant near room temperature.

\section{Harmonic and DTCF spectra}

Fig. 6 shows the comparison of the experimental $\mathrm{D}_{2}$-tagged IRPD spectrum of $\mathrm{SO}_{4}{ }^{2-}\left(\mathrm{H}_{2} \mathrm{O}\right)_{6}$ with simulated harmonic as well as $100 \mathrm{~K}$ DTCF spectra of the two isomers 6.9.3 and 6.12.0. Calculated IR spectra of other isomers are shown in the Fig. S2 of the ESI. $\dagger$ The scaled harmonic IR spectra for the two isomers (panels c and e in Fig. 6) are rather similar over the complete spectral range and both show similarities with the experimental spectrum. However, there are two differences, which favor an assignment to isomer 6.9.3. The most obvious is the prediction of five $\mathrm{O}-\mathrm{H}$ stretching bands in the range from $3200-3600 \mathrm{~cm}^{-1}$, in particular the intense band at $3263 \mathrm{~cm}^{-1}$, assigned to the hydrogen bonded $\left(\mathrm{ADD}-\mathrm{H}_{2} \mathrm{O} /\right.$ sulfate $) \mathrm{O}-\mathrm{H}$ stretching modes, in contrast to the single $\mathrm{O}-\mathrm{H}$ stretching band at 3525 in the harmonic spectrum of isomer 6.12.0. Note, the B3LYP-D3 calculations slightly underestimate the strength of the ADD- $\mathrm{H}_{2} \mathrm{O}$ /sulfate hydrogen bonds. The broader water bending band in the 6.9.3 spectrum also agrees better with the experimental spectrum. On the other hand, the harmonic spectrum of 6.9.3 predicts two water wagging modes of comparable IR intensity, of which only one is observed in the IRPD spectrum. This was one of arguments in favor of an assignment to isomer 6.12.0 in the original IRMPD study, which was based on measurements exclusively in the mid-IR spectral range $\left(540-1850 \mathrm{~cm}^{-1}\right){ }^{10}$

Additional insights can be gained from the results of the AIMD simulations (panels $b$ and d of Fig. 6). Compared to the harmonic IR spectrum the DTCF spectrum of the 6.9.3 isomer qualitatively improves the agreement with the experimental one, in particular because the AIMD simulation also accounts for temperature as well as dynamic aspects. ${ }^{17}$ The predicted 
a)

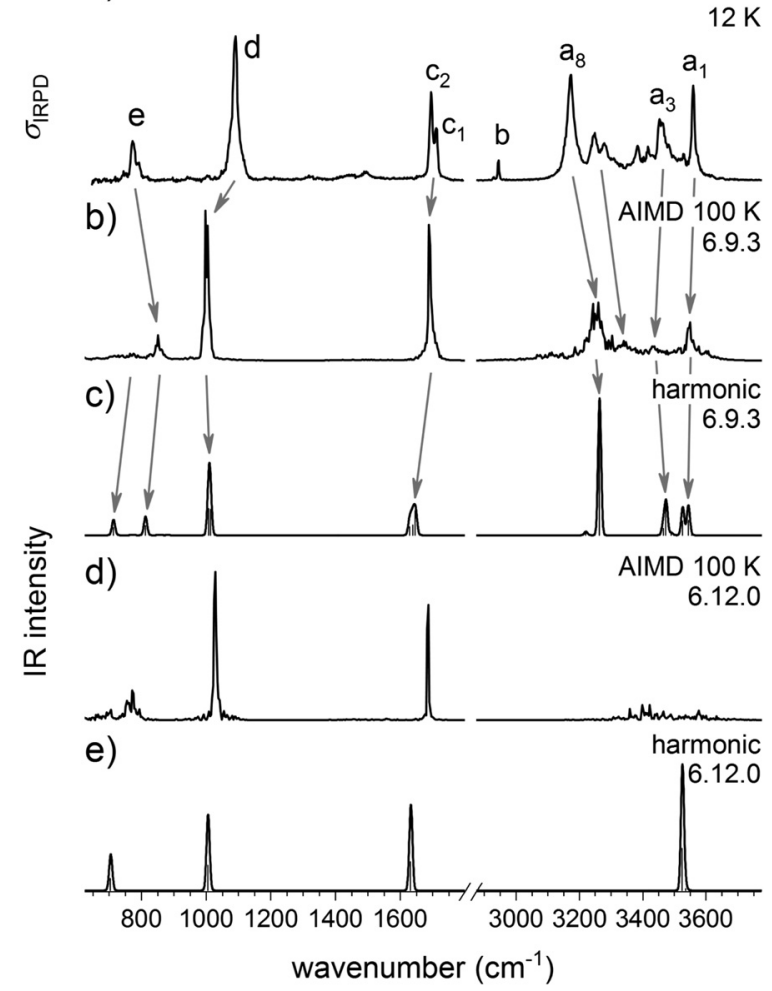

Fig. 6 Comparison of the IRPD spectra of $\mathrm{SO}_{4}{ }^{2-}\left(\mathrm{H}_{2} \mathrm{O}\right)_{6} \cdot \mathrm{D}_{2}$ (a) with computed anharmonic IR spectra from AIMD simulations $(b, d)$ and harmonic B3LYP/aug-cc-pVTZ IR spectra of bare $\mathrm{SO}_{4}{ }^{2-}\left(\mathrm{H}_{2} \mathrm{O}\right)_{6}$. Harmonic spectra are convoluted with a Gaussian line shape function (FWHM $=12 \mathrm{~cm}^{-1}$ ) and scaled by 0.960 .

absorption profile over the complete spectral range, but especially in the $\mathrm{O}-\mathrm{H}$ stretching region, captures the widths and relative intensities rather well, without the need for convolution as for the harmonic spectra. Moreover, the AIMD simulations predict only a single prominent band in the water wag region below $900 \mathrm{~cm}^{-1}$, in better agreement with experiment.
Unexpectedly, the results of the AIMD simulations for 6.12.0 also reveal an improved agreement compared to the harmonic IR spectrum. Instead of a single $\mathrm{O}-\mathrm{H}$ stretching peak, a $400 \mathrm{~cm}^{-1}$ broad, low intensity feature is predicted, suggesting that it may be difficult to rule out the presence of the 6.12.0 isomer, since its characteristic absorptions overlap with those of the 6.9.3 isomer. Moreover, these calculations suggest that the highest energy experimental band $\mathrm{a}_{1}$, previously assumed to contain significant contributions from isomer 6.12.0 based on the harmonic spectra, ${ }^{11}$ predominantly contains contributions from 6.9.3 (even if 6.12.0 were present). Summarizing, the current experimental IR(M)PD spectra are best rationalized by assuming the predominant presence of isomer 6.9.3, possibly with minor contributions from 6.12.0.

More detailed, atom-dependent information on the dynamic effects of the solvent molecules can be gained from the vibrational density of states (DOS) obtained by the Fourier transform of the velocity autocorrelation function for a particular atom, shown in Fig. 7. This shows that the $\mathrm{OH}$-stretch vibration of the DD- $\mathrm{H}_{2} \mathrm{O}$ covers a range of $300 \mathrm{~cm}^{-1}$. As demonstrated in a previous study on $\mathrm{HCO}_{3}{ }^{-}\left(\mathrm{H}_{2} \mathrm{O}\right),{ }^{35}$ the $\mathrm{DD}-\mathrm{H}_{2} \mathrm{O}$ is in an indifferent equilibrium since it's impossible to achieve a co-linear $\mathrm{O}-\mathrm{H} \cdots \mathrm{O}$ alignment for both hydrogen bonds, similar to the situation in $\mathrm{NO}_{3}{ }^{-}\left(\mathrm{H}_{2} \mathrm{O}\right){ }^{36}$ The DD- $\mathrm{H}_{2} \mathrm{O}$ can easily rock to one side, making one hydrogen bond shorter and better aligned, while sacrificing the other hydrogen bond. This motion leads to a broadening of the $\mathrm{OH}$ stretching and water wagging modes of the DD- $\mathrm{H}_{2} \mathrm{O}$ 's, which are particularly sensitive to the length of the hydrogen bond. In addition, the harmonic calculations confirm that the vibrations associated with this rocking motion are predicted at 72 and $107 \mathrm{~cm}^{-1}$, which coincides with the observed difference of $76 \mathrm{~cm}^{-1}\left(a_{7}-a_{8}\right)$ and $105 \mathrm{~cm}^{-1}\left(a_{6}-a_{8}\right)$. As a result, the IR intensity of the $\mathrm{DD}-\mathrm{H}_{2} \mathrm{O}$ wagging vibrations is smeared out over a broader spectral range $\left(\sim 150 \mathrm{~cm}^{-1}\right)$ due to the dynamic effects and therefore less apparent in the IRPD spectrum. In contrast, the rocking motion of the three ADD- $\mathrm{H}_{2} \mathrm{O}$ 's has a much smaller amplitude as a result of the

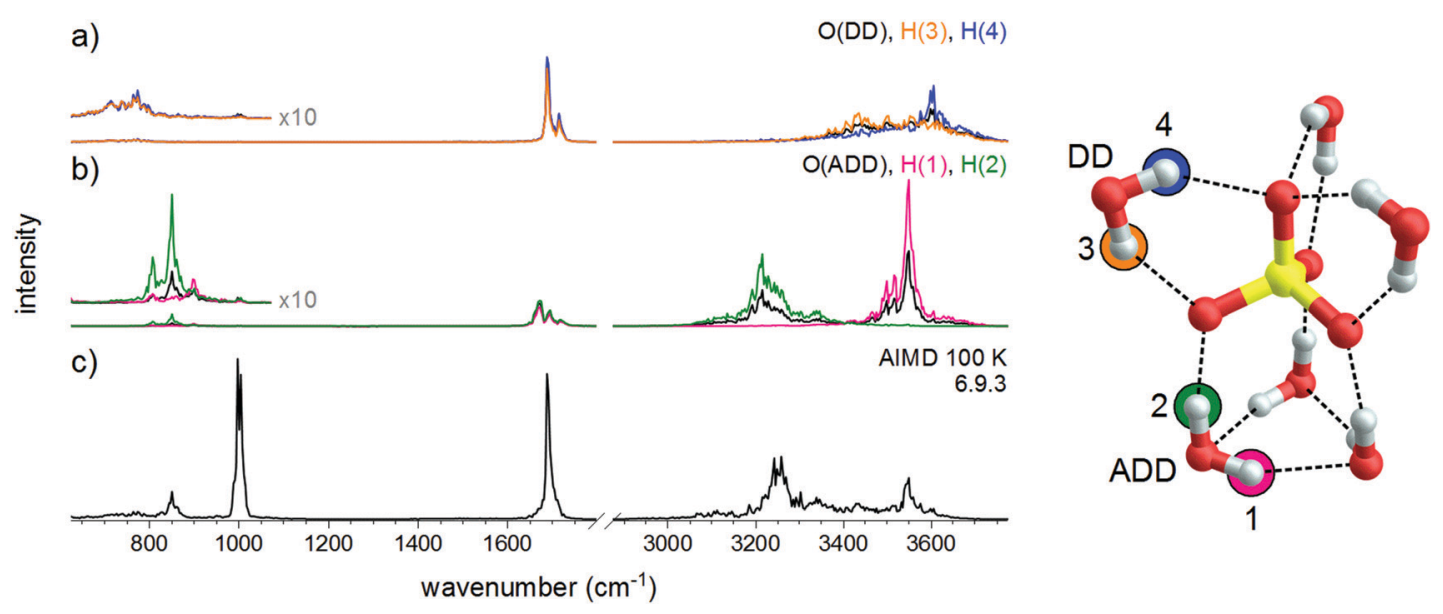

Fig. 7 Vibrational density of states plots for the $\mathrm{O}$ - and $\mathrm{H}$-atoms of the DD- $\mathrm{H}_{2} \mathrm{O}(\mathrm{a})$ and $\mathrm{ADD}-\mathrm{H}_{2} \mathrm{O}(\mathrm{b})$ hydrogen bonded water molecules based on the AIMD simulation of 6.9 .3 at $100 \mathrm{~K}$ (c). 
additional fixation by the third hydrogen bond. The DOS plots in Fig. 7 also support the assignment of bands $\mathrm{a}_{1}$ and $\mathrm{a}_{8}$ to $\mathrm{O}-\mathrm{H}$ stretches of the ADD- $\mathrm{H}_{2} \mathrm{O}$ 's hydrogen-bonded to a water molecule or the sulfate core, respectively. Finally, our calculations confirm that the three-membered water ring in 6.9.3 is stable over the whole simulation time, even when the simulation temperature is raised to $200 \mathrm{~K}$.

\section{Summary and conclusions}

The present study shows that predominantly a single spectroscopic species, namely the 6.9.3 and not the 6.12.0 isomer, is probed in IRPD experiments on $\mathrm{SO}_{4}{ }^{2-}\left(\mathrm{H}_{2} \mathrm{O}\right)_{6}$ as well as its fully deuterated isotopologue at temperatures up to $200 \mathrm{~K}$. While the vibrational signature of these two isomers is quite similar in the mid-IR region, in particular, when dynamic effects are considered, measurements in the $\mathrm{O}-\mathrm{H}(\mathrm{D})$ region prove to be more diagnostic, in this particular case, as was suggested previously. ${ }^{11,12}$ However, also in this spectral region the assignment is not as straight-forward as one would expect based on the harmonic simulations of the IR spectra, but anharmonic, temperature and tagging effects need to be considered as well and only the one- and two-color IRPD spectra obtained at cryogenic temperatures in combination with AIMD simulations allow for an unambiguous structural assignment.

The anharmonic spectra obtained from the AIMD simulations on $\mathrm{SO}_{4}{ }^{2-}\left(\mathrm{H}_{2} \mathrm{O}\right)_{6}$ accurately account for the most of the features across the IRPD spectrum. The simulations confirm the stability of the 6.9.3 isomer also at elevated temperatures $(\sim 200 \mathrm{~K})$ and show that the two types of water molecules (ADD vs. DD) behave quite differently. The position of the ADD waters are fixed by the ring, the DD waters (of both isomers) perform larger amplitude motion, leading to the broadening of the corresponding $\mathrm{O}-\mathrm{H}$ stretch and $\mathrm{H}_{2} \mathrm{O}$ wagging vibrations. Population of the 6.12.0 isomer is predicted to become favorable near room temperature and above. However, its spectral identification will be challenging, since it contains only DD water molecules, which are prone to large amplitude motion and hence lead to particularly broad features in the vibrational spectrum, especially at $300 \mathrm{~K}$. Nonetheless, the insights obtained in the present study should prove helpful to reliably assign structures of sulfate-water clusters of different size.

\section{Conflicts of interest}

There are no conflicts to declare.

\section{Acknowledgements}

This work has been supported by the German Research Foundation (DFG) as part of the individual research Grant No. AS133/3-1 "Spectroscopic Characterization of Salt Dissolution in Microhydrated Cluster Ions and at the Water/Vapor Interface." ZFL thanks the support from the National Natural Science Foundation of China (Grant No. 21473151) and from CUHK through a Direct Grant (3132676). The authors are grateful to Alexandra Giermann (Universität Leipzig) for her assistance in preparing and handling sulfuric acid solutions. K. R. A. acknowledges instrumental support from the Fritz-HaberInstitute of the Max-Planck Society.

\section{References}

1 V. Ramanathan, P. J. Crutzen, J. T. Kiehl and D. Rosenfeld, Science, 2001, 294, 2119-2124.

2 A. Lee, P. A. Dawson and D. Markovich, Int. J. Biochem. Cell Biol., 2005, 37, 1350-1356.

3 S. W. Squyres, J. P. Grotzinger, R. E. Arvidson, J. F. Bell, W. Calvin, P. R. Christensen, B. C. Clark, J. A. Crisp, W. H. Farrand, K. E. Herkenhoff, J. R. Johnson, G. Klingelhofer, A. H. Knoll, S. M. McLennan, H. Y. McSween, R. V. Morris, J. W. Rice, R. Rieder and L. A. Soderblom, Science, 2004, 306, 1709-1714.

4 Y. J. Zhang and P. S. Cremer, Curr. Opin. Chem. Biol., 2006, 10, 658-663.

5 A. T. Blades and P. Kebarle, J. Am. Chem. Soc., 1994, 116, 10761-10766.

6 W. R. Cannon, B. M. Pettitt and J. A. McCammon, J. Phys. Chem., 1994, 98, 6225-6230; X. B. Wang, X. Yang and L. S. Wang, Int. Rev. Phys. Chem., 2002, 21, 473-498.

7 X. B. Wang, J. B. Nicholas and L. S. Wang, J. Chem. Phys., 2000, 113, 10837-10840.

8 C. C. Pye and W. W. Rudolph, J. Phys. Chem. A, 2001, 105, 905-912.

9 R. L. Wong and E. R. Williams, J. Phys. Chem. A, 2003, 107, 10976-10983; C. G. Zhan, F. Zheng and D. A. Dixon, J. Chem. Phys., 2003, 119, 781-793; B. Gao and Z. F. Liu, J. Chem. Phys., 2004, 121, 8299-8306; B. Gao and Z. F. Liu, J. Chem. Phys., 2005, 123, 224302.

10 J. Zhou, G. Santambrogio, M. Brümmer, D. T. Moore, G. Meijer, D. M. Neumark and K. R. Asmis, J. Chem. Phys., 2006, 125, 111102.

11 M. F. Bush, J. T. O’Brien, J. S. Prell, R. J. Saykally and E. R. Williams, J. Am. Chem. Soc., 2007, 129, 1612-1622.

12 X. B. Wang, A. P. Sergeeva, J. Yang, X. P. Xing, A. I. Boldyrev and L. S. Wang, J. Phys. Chem. A, 2009, 113, 5567-5576.

13 D. S. Lambrecht, G. N. Clark, T. Head-Gordon and M. HeadGordon, J. Phys. Chem. A, 2011, 115, 5928-5935.

14 D. S. Lambrecht, L. McCaslin, S. S. Xantheas, E. Epifanovsky and M. Head-Gordon, Mol. Phys., 2012, 110, 2513-2521.

15 N. Mardirossian, D. S. Lambrecht, L. McCaslin, S. S. Xantheas and M. Head-Gordon, J. Chem. Theory Comput., 2013, 9, 1368-1380.

16 A. T. Blades, J. S. Klassen and P. Kebarle, J. Am. Chem. Soc., 1995, 117, 10563-10571; A. T. Blades and P. Kebarle, J. Phys. Chem. A, 2005, 109, 8293-8298.

17 L. Jiang, S.-T. Sun, N. Heine, J.-W. Liu, T. I. Yacovitch, T. Wende, Z.-F. Liu, D. M. Neumark and K. R. Asmis, Phys. Chem. Chem. Phys., 2014, 16, 1314-1318.

18 R. A. Relph, B. M. Elliott, G. H. Weddle, M. A. Johnson, J. Ding and K. A. Jordan, J. Phys. Chem. A, 2009, 113, 
975-981; N. Heine, M. R. Fagiani, M. Rossi, T. Wende, G. Berden, V. Blum and K. R. Asmis, J. Am. Chem. Soc., 2013, 135, 8266-8273.

19 N. Heine and K. R. Asmis, Int. Rev. Phys. Chem., 2014, 34, 1-34; N. Heine and K. R. Asmis, Int. Rev. Phys. Chem., 2016, 35, 507.

20 M. Brümmer, C. Kaposta, G. Santambrogio and K. R. Asmis, J. Chem. Phys., 2003, 119, 12700-12703; D. J. Goebbert, T. Wende, R. Bergmann, G. Meijer and K. R. Asmis, J. Phys. Chem. A, 2009, 113, 5874-5880.

21 W. R. Bosenberg and D. R. Guyer, J. Opt. Soc. Am. Soc. B, 1993, 10, 1716-1722.

22 M. J. Frisch, G. W. Trucks, H. B. Schlegel, G. E. Scuseria, M. A. Robb, J. R. Cheeseman, G. Scalmani, V. Barone, G. A. Petersson, H. Nakatsuji, X. Li, M. Caricato, A. V. Marenich, J. Bloino, B. G. Janesko, R. Gomperts, B. Mennucci, H. P. Hratchian, J. V. Ortiz, A. F. Izmaylov, J. L. Sonnenberg, D. Williams, F. Ding, F. Lipparini, F. Egidi, J. Goings, B. Peng, A. Petrone, T. Henderson, D. Ranasinghe, V. G. Zakrzewski, J. Gao, N. Rega, G. Zheng, W. Liang, M. Hada, M. Ehara, K. Toyota, R. Fukuda, J. Hasegawa, M. Ishida, T. Nakajima, Y. Honda, O. Kitao, H. Nakai, T. Vreven, K. Throssell, J. A. Montgomery Jr., J. E. Peralta, F. Ogliaro, M. J. Bearpark, J. J. Heyd, E. N. Brothers, K. N. Kudin, V. N. Staroverov, T. A. Keith, R. Kobayashi, J. Normand, K. Raghavachari, A. P. Rendell, J. C. Burant, S. S. Iyengar, J. Tomasi, M. Cossi, J. M. Millam, M. Klene, C. Adamo, R. Cammi, J. W. Ochterski, R. L. Martin, K. Morokuma, O. Farkas, J. B. Foresman and D. J. Fox, Gaussian 16, Revision B.01, Gaussian, Inc., Wallingford, CT, 2016.

23 A. D. Becke, J. Chem. Phys., 1993, 98, 5648-5652.

24 T. H. Dunning, J. Chem. Phys., 1989, 90, 1007-1023; D. E. Woon and T. H. Dunning, J. Chem. Phys., 1993, 98, 1358-1371.
25 S. Grimme, S. Ehrlich and L. Goerigk, J. Comput. Chem., 2011, 32, 1456-1465.

26 J. Bloino and V. Barone, J. Chem. Phys., 2012, 136, 124108.

27 J. VandeVondele, M. Krack, F. Mohamed, M. Parrinello, T. Chassaing and J. Hutter, Comput. Phys. Commun., 2005, 167, 103-128.

28 G. Lippert, J. r. Hutter and M. Parrinello, Theor. Chem. Acc., 1999, 103, 124-140.

29 S. Goedecker, M. Teter and J. Hutter, Phys. Rev. B: Condens. Matter Mater. Phys., 1996, 54, 1703-1710; C. Hartwigsen, S. Goedecker and J. Hutter, Phys. Rev. B: Condens. Matter Mater. Phys., 1998, 58, 3641-3662; M. Krack, Theor. Chem. Acc., 2005, 114, 145-152.

30 A. D. Becke, Phys. Rev. A: At., Mol., Opt. Phys., 1988, 38, 3098-3100; C. Lee, W. Yang and R. G. Parr, Phys. Rev. B: Condens. Matter Mater. Phys., 1988, 37, 785-789.

31 G. J. Martyna and M. E. Tuckerman, J. Chem. Phys., 1999, 110, 2810-2821.

32 S. Nose, J. Chem. Phys., 1984, 81, 511-519.

33 D. A. McQuarrie, Statistic Mechanics, Harper-Collins Publishers, New York, 1976.

34 P. J. Brannon, C. H. Church and C. W. Peters, J. Mol. Spectrosc., 1968, 27, 44-54.

35 X. Kong, S.-T. Sun, L. Jiang and Z.-F. Liu, Phys. Chem. Chem. Phys., 2018, 20, 4571-4578.

36 X. B. Wang, X. Yang, L. S. Wang and J. B. Nicholas, J. Chem. Phys., 2002, 116, 561-570; D. J. Goebbert, E. Garand, T. Wende, R. Bergmann, G. Meijer, K. R. Asmis and D. M. Neumark, J. Phys. Chem. A, 2009, 113, 7584-7592; N. Heine, E. G. Kratz, R. Bergmann, D. P. Schofield, K. P. Asmis, K. D. Jordan and A. B. McCoy, J. Phys. Chem. A, 2014, 118, 8188-8197. 